\title{
Family building in parents with Down's syndrome children
}

\author{
A R BOON* \\ From the Regional Genetics Advisory Service, Newcastle upon Tyne
}

SUMMARY Fertility and contraceptive practice were examined by interview with parents of 100 Down's syndrome patients. Before the birth of the affected child, fertility was low by comparison with that in married women in the general population, and to this the socioeconomic class bias in the sample is thought to be contributory. By comparison with the number of children intended had the Down's syndrome propositus been unaffected, the actual subsequent fertility was reduced. There seems to be an increased number of other types of defect in these sibships. As a contraceptive method, sterilisation was chosen by an elevated proportion of parents. Despite their acceptance of the child, the majority of mothers would wish to terminate any future pregnancy in which an abnormal fetus was diagnosed.

The birth of a child with Down's syndrome is an indescribable shock to the parents. It would not be surprising if this altered their whole attitude to further family building; one might expect their contraceptive practices subsequently to be particularly efficient, and one might expect their attitude to pregnancy termination to differ from those of the population at large. Yet there is little information in the literature on these and related questions. To provide it, a survey was made in a sample of parents with Down's syndrome children in the north-east of England.

\section{Materials and methods}

A survey was conducted in 1978-79 in the northern hospital region which, centred in Newcastle upon Tyne, extends from Berwick in the north to the Cleveland area in the south, and to Whitehaven in the west. One hundred families agreed to cooperate in the study. Seventy-one of them had been referred to the Genetics Advisory Service for chromosome examination of the affected child by the family doctor or paediatrician. Of these, 34 pairs of parents who were concerned at the risk of recurrence in a future pregnancy or in the children of their normal sons and daughters subsequently received genetic counselling through the Advisory Service; the other 37 were counselled by the paediatrician, or regarded their families as complete and did not request genetic

*Present address: 1 Osborne Villas, Osborne Avenue, Newcastle upon Tyne NE2 $1 \mathrm{LJ}$. advice. There were a further 15 families who were members of the local Down's Syndrome Association, and 14 from the Hartlepool community health register, none of whom had been previously referred for genetic counselling.

General practitioners, or consultant paediatricians, were informed by letter about the? survey, and their agreement was requested before a family interview. Confirmation that the Down's child was alive and living with the parents was obtained from the family doctor. All families were visited at home by appointment, the interviews being carried out by either the author or a health visitor from the Genetics Advisory Service. The interview, which usually lasted from one to two hours, sometimes longer, was structured, and a standard questionnaire was used.

\section{KARYOTYPES}

There were 62 male and 38 female Down's syndrome cases, giving a sex ratio of $1 \cdot 6$ (table 1$)$. Chromosome analysis was carried out on all but two of the children, whose parents refused permission to take a blood specimen. Both cases, one male and one female, were severely handicapped children born to older mothers. Among the Down's syndrome patients there was one female de novo 14/21 translocation and one male de novo $21 / 21$ translocation. In addition, there were four mosaics, and the remaining 90 had trisomy 21. Karyotypes in the younger parents were also analysed. One balanced D/G $(14 / 21)$ translocation carrier was detected from 50 
Table 1 Chromosome analysis of Down's syndrome cases

\begin{tabular}{lllllll}
\hline & Nondisjunction & & $\begin{array}{l}\text { Parental } \\
14 / 21\end{array}$ & $\begin{array}{l}\text { Normal parents } \\
\text { child 14/21 } \\
\text { translocation }\end{array}$ & $\begin{array}{l}\text { Normal parents } \\
\text { child 21/21 } \\
\text { translocation }\end{array}$ & Total \\
\hline chromosome 21 & Mosaic & 36 translocation & 1 & 0 & 1 & 61 \\
Female & 34 & 1 & 1 & 1 & 0 & 37 \\
Totals & 90 & 4 & 2 & 1 & 1 & 98 \\
\hline
\end{tabular}

fathers tested, and one 14/21 translocation from 54 mothers.

THE PARENTS

The mean maternal age (at previous birthday) at the birth of the Down's syndrome child was $30 \cdot 3$ years, ranging from 18 to 46 , with a standard deviation of $7 \cdot 01$. The corresponding mean paternal age was $32 \cdot 1$ years, ranging from 18 to 53 , and with a standard deviation of $7 \cdot 62$. Though with a mean age difference between the parents of 1.8 years, the paternal age was closely correlated with that of the mother; the shift towards the older age group appeared slightly more pronounced in fathers (figs $1 \& 2$ ). The distribution of the maternal ages at the birth of the Down's syndrome child clearly suggests a bimodal distribution. Comparing this with that expected from the Registrar-General's ${ }^{1}$ maternal age distribution shows a clear preponderance of older mothers.

Social class (table 2) was assigned by father's occupation according to the Registrar-General's ${ }^{1}$ classification and compared with the distribution for the region in the 1971 census. There is clearly a preponderance of parents in social classes 1 and 2 $\left(x^{2}{ }_{3}=17 \cdot 3\right)$ and there are several possible explanations for this. Firstly, these are the parents
Table 2 Distribution of families by socioeconomic class

\begin{tabular}{llllll}
\hline & \multicolumn{3}{l}{ Class } & & \\
\cline { 2 - 6 } & $I$ & $I I$ & $I I I$ & $I V$ & $V$ \\
\hline No. observed & 8 & 27 & 47 & 12 & 6 \\
No. expected & 3.78 & 15.87 & 50.58 & 17.12 & 12.65 \\
\hline
\end{tabular}

most likely to request genetic counselling. Secondly, these mothers tend to marry later than in the lower socioeconomic groups, and indeed the average age at marriage of the mothers of social classes 1 and 2 in this sample was 34 years. Thirdly, they tend to delay starting a family until they have established a secure and comfortable home.

\section{Results}

Maternal fertility before the birth of the Down's syndrome child is set out in table 3 . There is a highly significant departure from the parities expected from the Registrar-General's distribution for married women $\left(\chi^{2} s=73.6\right)$ due to a pronounced excess of nulliparous women and deficit of those with more than one child, presumably for the reasons given above. When the expected parities are adjusted to

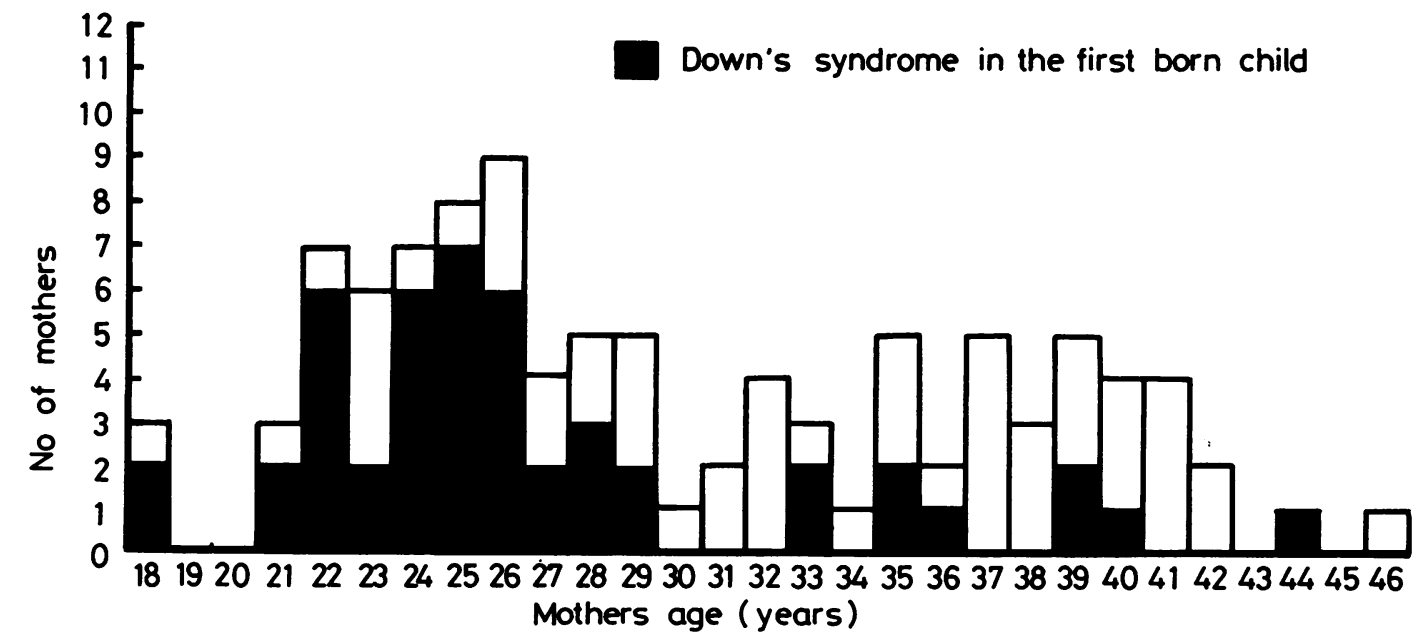

Fig 1 Age of mother at birth of Down's syndrome child. 


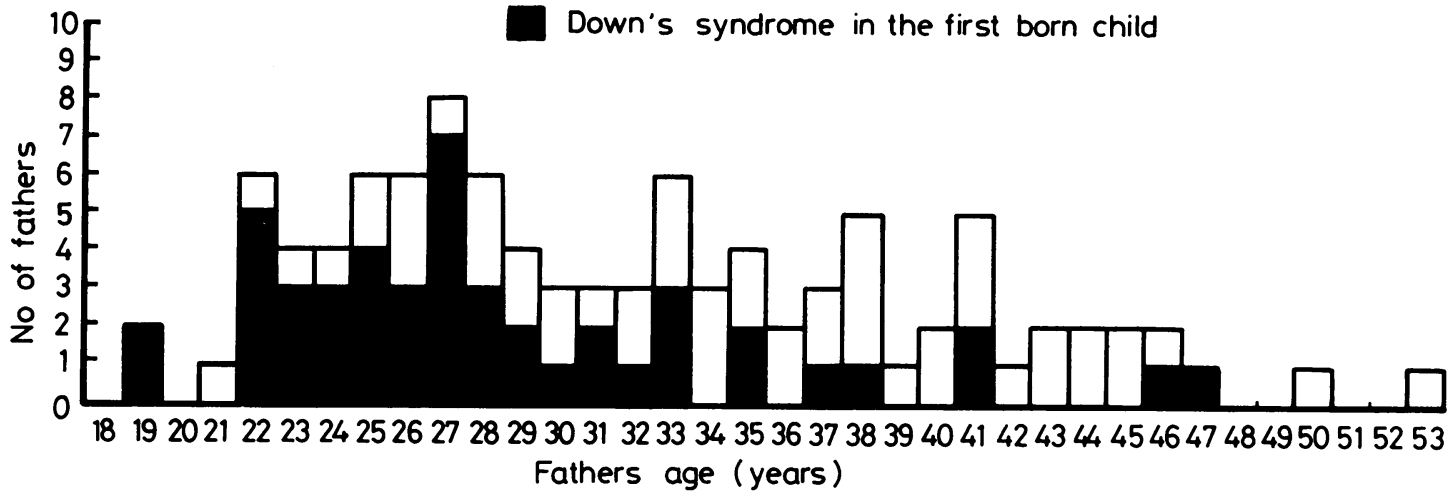

Fig 2 Age of father at birth of Down's syndrome child.

Table 3 Parity before birth of Down's syndrome child

\begin{tabular}{|c|c|c|c|}
\hline \multirow[b]{2}{*}{ Parity } & \multirow{2}{*}{$\begin{array}{l}\text { Observed } \\
\text { number of } \\
\text { females }\end{array}$} & \multicolumn{2}{|c|}{ Expected number } \\
\hline & & Unweighted & $\begin{array}{l}\text { Weighted by } \\
\text { maternal age }\end{array}$ \\
\hline 0 & 47 & $16 \cdot 2$ & $19 \cdot 5$ \\
\hline 1 & 23 & $23 \cdot 6$ & 19.6 \\
\hline 2 & 16 & $32 \cdot 2$ & $31 \cdot 6$ \\
\hline 3 & 8 & $15 \cdot 8$ & $16 \cdot 6$ \\
\hline 4 & 3 & 6.9 & $8 \cdot 0$ \\
\hline 5 & 1 & $2 \cdot 8$ & $3 \cdot 5$ \\
\hline 6 & 0 & $1 \cdot 3$ & \\
\hline 7 & 1 & 0.6 & \\
\hline 8 & 0 & $0 \cdot 3$ & $1 \cdot 2$ \\
\hline 9 & 1 & $0 \cdot 1$ & \\
\hline
\end{tabular}

take account of the maternal age distribution, the same pattern remains $\left(\chi^{2}{ }_{5}=55 \cdot 3\right)$.

For their age, then, the mothers at the birth of the index cases tend to be of lower parity than the population at large. Examining further their past reproductive histories, before the birth of the Down's child the mothers had had 147 pregnancies, of which 112 had resulted in a live birth. This number is considerably lower than that expected from the Registrar-General's tabulations (172.3) for a sample of married women with the same age distribution. The number of spontaneous abortions (29) appears to be rather high, and the two translocation carrier parents seem to have made little contribution to this. The number of stillbirths also appears slightly higher than expected, but this is due to the inclusion of two pregnancies which produced premature twins. One singleton stillbirth was of a male with spina bifida, the other, a female, was due to toxaemia. The mothers of male and female Down's children are very similar in their reproductive histories.

After the birth of the index case, there is a distinct lack of children born. Altogether there were 67 pregnancies. Two were terminated after amniocentesis, one of a Down's fetus to a mother carrying a 14/21 translocation, and the other of a twin pregnancy where one twin had a large exomphalos (carried out in 1976, after an elevated AFP result but before a high resolution ultrasound scan was? available). There were eight spontaneous abortions $\stackrel{O}{\circ} \overrightarrow{f r}$ and two pregnancies ended in stillbirths, again of twins. After the birth of the Down's child, 55 liveborn몸 children were produced in these families.

The mothers were asked about their family-building intentions. Once the affected child 0 had been born, 61 of the mothers would certainly $\overrightarrow{0}$ have liked more children, three were unable to say, $\infty$ but 36 decided not to add to their families because they thought that they were too old or that they could not cope on account of the affected child, or that they had decided that their family was already complete. As regards the number of children previously intended if the propositus had been a normal child, 24 mothers had planned for one more child, 18 for two more, 3 for three more, and 4 for four more, while 51 could not say. These last estimates, excluding the half of the sample who could not answer, show previous intentions of a minimum of 85 more children. By comparison with this, the 55 achieved livebirths appears to represent a distinct restriction of reproduction.

Ninety-one mothers had never considered the possibility that they would produce an abnormal child, the others having obtained their knowledge from the press (2), from radio or television (3), and from acquaintanceship with other families with a Down's child (4). Just over half the mothers regarded themselves as too old to contemplate further children, or regarded their family as complete, and so were not concerned to seek advice about recurrence risks. The remaining 49 sought advice, 34 being referred to the Genetics Advisory Service, 14 being 
counselled by a family doctor or the consultant paediatrician, while one was erroneously advised a $50 \%$ recurrence risk by a nurse.

Twenty of the children following the propositus were born before 1976, when prenatal diagnosis first became available as a service in the Region. In the 35 pregnancies after 1976, five mothers were not offered the reassurance of antenatal tests, and four mothers refused amniocentesis for religious reasons. Twenty-four mothers, following genetic counselling, decided to add to their families, two mothers had accidental pregnancies, and all 26 availed themselves of amniocentesis.

These decisions were not, of course, made solely on the basis of the presence of the Down's syndrome child. Other problems occurred in these families. Of the 169 liveborn brothers and sisters of the index cases, one brother was mentally retarded, one girl had Apert's syndrome, and one sister had congenital cataracts. One brother had a diaphragmatic hernia, and a sister had congenital dislocation of the hips. Two siblings died soon after birth, one girl with Holt-Oram syndrome, and one premature male with respiratory distress syndrome. Thus, seven of the 169 liveborn siblings had serious congenital defects, a figure slightly higher than expected.

To account for the restriction of fertility subsequent to the birth of the Down's child, contraceptive methods employed must have been reasonably efficient. Many couples used more than one method (table 4). In 26 couples, a quarter of the families in the study, sterilisation was requested by one of the parents after the Down's syndrome child was born. In three cases this was done without seeking informed advice about genetic risks, and in one case the parents had erroneously been advised a high risk of recurrence. One mother became pregnant with an IUCD in situ and she subsequently aborted spontaneously. There were 20 Roman Catholic couples in the study, six of whom produced four or more children. They were reluctant to practise contraception, but ultimately five parents (four fathers and one mother) agreed to sterilisation.

The attitudes of the mothers to induced abortion for genetic reasons were interesting. Certainly the majority of parents had accepted the Down's child

Table 4 Methods of contraception

\begin{tabular}{lc}
\hline Method used & No. of users \\
\hline Contraceptive pill & 33 \\
Condom & 25 \\
IUCD & 13 \\
Diaphragm & 3 \\
Sterilised (tubal ligation 14; vasectomy 12) & 26 \\
Rhythm & \multicolumn{2}{|c}{9} \\
Coitus interruptus \\
Nil
\end{tabular}

over a period of months or years, and some of them admitted that the affected child had brought much love and affection into the family. Yet 48 mothers out of 71 stated that they would agree to terminate a pregnancy if antenatal diagnosis proved the fetus to be affected. Six would refuse a termination of pregnancy on religious, and 13 on moral and psychological, grounds. There were four mothers who were uncertain concerning their attitude towards pregnancy termination. Having accepted the handicapped child, they realised that there were varying degrees of mental handicap, and that no one could predict before birth how severely affected the child might be.

\section{Discussion}

It must be accepted that the cases in this survey were not a random sample of parents of Down's syndrome children, and therefore bias may have affected the results. Nevertheless a number of points of interest are suggested.

The importance of the maternal age factor in the production of Down's syndrome has been recognised since $1909 .{ }^{2}$ Several recent population studies show changes in the incidence and parental age at the birth of the Down's syndrome child. In Liverpool, Owens et $\mathrm{al}^{3}$ found that the mean maternal age at the birth of the Down's syndrome child fell from 36.7 to 29.0 years between 1961 and 1979. This was attributable to the reduction in live births to women over the age of 40 years. In 233 Down's syndrome births in Manitoba, $1965-74,{ }^{4}$ the mean maternal age declined for all live births from $27 \cdot 2$ years in 1960 to $25 \cdot 1$ years in 1974 , and in the same period the mean maternal age for Down's syndrome births fell from 34.8 years to 30.6 years. Similarly, in Japan the mean maternal age for Down's syndrome fell from 31.3 in 1950 to 29.6 in $1973 ;^{5}$ they suggest that there has been no change in the age-specific incidences of Down's syndrome in Japan. In Copenhagen, Mikkelsen $e a^{6}{ }^{6}$ reported that in a control sample of normal live births $23.4 \%$ were to women over 30 in 1960 , and this decreased to $16 \cdot 2 \%$ by 1971 . There was a corresponding decrease in the Down's syndrome births from $52 \cdot 6 \%$ to $40 \%$. However, $\mathrm{Uchida}^{7}$ found a small tendency for the incidence of Down's syndrome in younger mothers to increase and suggested that environmental factors might be the cause of this.

There is conflicting evidence regarding the paternal age effect in chromosome disorders. Since the father's age is usually correlated with that of the mother, it proved difficult to attribute the defect to the father by simple epidemiological methods. Proof that the father was responsible had to await the 
development of laboratory methods to distinguish the origin of the additional chromosome. Hanson and Mikkelsen ${ }^{8}$ demonstrated that $27 \%$ nondisjunction trisomy 21 may be paternal in origin, and a figure of $14 \%$ was calculated by Roberts and Callow. ${ }^{9}$ But data are still too few to establish that the paternal nondisjunction trisomy 21 is related to age. Hook and Cross $^{10}$ were unable to show an association between paternal age and Down's syndrome in 98 cases of prenatal diagnosis. Similarly, in British Columbia, Hook et al ${ }^{11}$ showed no significant age effect. However, in Norway Erickson and Bjerkedal ${ }^{12}$ demonstrated a significant age effect in fathers over 50 where control for maternal age was taken into account. Stene $e a^{13}$ also demonstrated that in fathers over 44 years there was an elevated risk for Down's syndrome fetuses. The elevated mean paternal and maternal ages and the bimodal distribution of the latter indicate the relevance of parental age in the aetiology of Down's syndrome in the present data. However, there seem also to be other biosocial factors. First is the lower fertility before the birth of the Down's syndrome child. This recalls the opinion of Jenkins ${ }^{14}$ that Down's syndrome sibships tended to be smaller than usual, though Penrose ${ }^{15}$ found no such reduction in his Colchester survey where there averaged 5.8 sibs for Down's patients. While the present finding of reduced previous fertility seems more likely to be attributable to the nature of the Down's syndrome sample, with the deferment of reproduction it may also indicate some biological factor. The apparent elevated number of sibs with other defects suggests that these parents may in some biological way differ slightly from others. Certainly this feature requires further investigation in other families with a Down's syndrome child.

Many of the couples referred for genetic counselling were young and anxious to increase the number of children in the family. With 47 of the Down's syndrome cases being the first born in the family, the apparent deficit of later children represents a reluctance to have further children on the part of some, and the youth of others who are likely to add to their families after genetic counselling. A proportion of those from the professional classes who were in the older age group (35-40) were aware of the genetic counselling service and concerned about recurrence risks, not only for themselves but also for other family members. Zorzi et $a l,{ }^{18}$ in a postal survey to assess adequacy of genetic counselling services, found that the results were biased in favour of the better educated and more self-motivated individuals. Oetting and Steele,${ }^{17}$ in assessing results of genetic counselling of a group of 23 pairs of Down's syndrome parents in the United
States matched with a similar group of non-counselled parents, noted that parents with a better education and in the higher socioeconomic groups tend to use counselling services more frequently than others. Subsequent reproduction in the two groups in the United States survey was almost identical, 9 liveborn infants in each group, of which 17 were healthy. There was a total of 12 spontaneous abortions, higher than expected, and two terminations without the benefit of amniocentesis. In the present study there were eight early spontaneous abortions following the Down's syndrome child (29) which was higher than the accepted level in the general population.

Details of methods of contraception are not well documented. While a $1 \%$ recurrence risk of Down's syndrome due to nondisjunction is low in genetic terms, the presence of a mentally handicapped child is clearly a burden for the parents, indicated by the numbers seeking sterilisation of one partner: 13 out of $46(28 \%)$ in the Oetting and Steele survey, ${ }^{17}$ very close to the 26 out of $100(26 \%)$ reported here. Sterilisation is clearly a more frequent option among $\underset{T}{0}$ iv Down's syndrome parents as compared with $100 \stackrel{\circ}{\vec{D}}$ mothers who produced a child with Fallot's tetralogy, ${ }^{18}$ of whom only seven couples requested $\stackrel{\circ}{2}$ sterilisation; the difference is possibly due to the fact $\vec{c}$ that in the latter group the handicap in a child is physical, which in the majority of cases can be cured유 or alleviated by surgery.

The request for and acceptance of prenata diagnosis by amniocentesis in the present study, where 26 mothers derived reassurance of a chromosomally normal infant after the Down's syndrome child, was more popular than in the study of Oetting and Steele in the United States where only three of the 18 such couples availed themselves of amniocentesis. It is unclear whether this was due to financial constraint or to the religious or moral convictions of the parents, or to a different interpretation of the balance of the $1 \%$ recurrence risk for Down's syndrome against the $1 \%$ risk of causing an accidental miscarriage by amniocentesis. Most mothers feel greatly reassured on finding that the infant has a normal karyotype. Not all, however, appreciate that amniocentesis does not exclude the presence of other major congenital abnormalities in subsequent children.

Smith $^{19}$ suggested that amniocentesis should be offered to all pregnant mothers, since most Down's syndrome children are born to mothers between 20 and 30 years of age. Certainly women in this age group account for the largest total number of births and produce the highest number of Down's babies but their incidence is much lower than in the older 
mothers, and this suggestion is not possible practically and economically.

In the past the level of educability of Down's syndrome children was frequently underestimated by families and professionals. ${ }^{20}$ That parents should be encouraged to stimulate their Down's children from a very early age in order to elicit their full potential was clearly appreciated by the mothers in this study. They also were aware of the wide range of mental handicap in Down's syndrome children, their capacity for integration into the community, and the dramatic improvement of their expectation of life by the advent of antibiotics for the treatment of recurrent infections, and improved heart surgery for the correction of congenital heart defects which may occur in $30 \%$ of cases. But many parents look ahead to the time when they wilı no longer be present to look after the affected children.

Many Down's syndrome children are happy and lovable individuals and become fully integrated into the family, especially in the larger sibships where acceptance seems easier. However, there are other implications. That parents appreciate these was shown by the finding that 48 out of 71 mothers of Down's syndrome children would be agreeable to the interruption of a further affected pregnancy.

\section{Conclusions}

Thus it appears that the birth of a child with Down's syndrome restricts the family building that was originally intended. There is a slight suggestion of an increased number of other types of defect in these families which may also influence such decisions. The contraceptive practice seems to have been efficient, and the option of sterilisation chosen more frequently than in a comparable series of families where the child's defect was not accompanied by mental handicap. Awareness of the problems of coping with the handicapped child, and those likely to arise in the future, is thought to be a contributory factor in the proportion of mothers who would approve the termination of pregnancy where the fetus was shown to be affected.

The results indicate, firstly, that it is desirable that all children with Down's syndrome should be karyotyped, including those born to the older mother, so that those families with a hereditary translocation may be aware of the high recurrence risk for siblings and other more remote relatives who may carry it. Secondly, even in this sample with its slight bias in composition, several parents had received erroneous advice concerning recurrence risks, which affected their subsequent actions. To prevent this, formal genetic counselling should be available for all families with a Down's syndrome child, and contraception, sterilisation, the availability and procedure of amniocentesis and other more recent developments discussed, together with the parental attitude to pregnancy termination. Discussion of the educability potential for an affected child and encouragement of the parents are also essential at this time.

Thanks are due to Professor D F Roberts for help and advice in producing this paper; to Mrs D Gibson and Mrs $J$ Le Gassicke who assisted with the visiting of these families; to Mrs A Stephenson for help with the statistical analysis; and to the Marie Stopes Research Fund for providing a grant to assist with the cost of collating the data.

\section{References}

${ }^{1}$ Registrar-General. Classification of occupation. London: HMSO, 1971.

'Shuttleworth GE. Mongolian imbecility. Br Med J 1909; 2: 661 .

${ }^{3}$ Owens JR, Harris F, Walker S, McAllister E, West L. The incidence of Down's syndrome over a 19-year period with special reference to maternal age. $J$ Med Genet 1983; 20: $90-3$.

4Evans JA, Hunter AGW, Hamerton JL. Down's syndrome and recent demographic trends in Manitoba.J Med Genet 1978; 15: 43-7.

${ }^{5}$ Kuroki Y, Yamamoto Y, Matsui I, Kurita T. Down's syndrome and maternal age in Japan, 1950-1973. Clin Genet 1977; 12: 43-6.

${ }^{6}$ Mikkelsen M, Fischer G, Stene J, Stene E, Petersen E. Incidence study of Down's syndrome in Copenhagen, 1960-1971: with chromosome investigation. Ann Hum Genet 1976; 40: 177-82.

${ }^{7}$ Uchida IA. Epidemiology of mongolism: the Manitoba study. Ann NY Acad Sci 1970; 171: 361-9.

'Hansson A, Mikkelsen M. The origin of the extra chromosome 21 in Down syndrome: studies of fluorescent variants and satelite association in 26 informative families. Cytogenet Cell Genet 1978; 20: 194-203.

${ }^{9}$ Roberts DF, Callow MH. Origin of the additional chromosome in Down's syndrome: a study of 20 families. J Med Genet 1980; 17: 363-7.

${ }^{10}$ Hook EB, Cross PK. Paternal age and Down's syndrome genotypes diagnosed prenatally: no association in New York State data. Hum Genet 1982; 62: 167-74.

${ }^{1}$ Hook EB, Cross PK. Lamson SH, Regal RR, Baird PA, Uh SH. Paternal age and Down syndrome in British Columbia. Am J Hum Genet 1981; 33: 123-8.

${ }^{12}$ Erickson JD, Bjerkedal T. Down syndrome associated with father's age in Norway. $J$ Med Genet 1981; 18: 22-8.

${ }^{13}$ Stene J, Stene E, Stengel-Rutkowski S, Murken JD. Paternal age and Down's syndrome: data from prenatal diagnosis (DFG). Hum Genet 1981; 59: 119-24.

14 Jenkins RL. Etiology of mongolism. Am J Dis Child 1933; 45: 506.

${ }^{15}$ Penrose LS. The biology of mental defect. London: Sidgwick \& Jackson. 
${ }^{16}$ Zorzi G, Thurman SK, Kistenmacher ML. Importance and adequacy of genetic counselling information: impression of parents with DS children. Mental Retardation 1980 (Oct); 225.

${ }^{17}$ Oetting LAS, Steele MW. A controlled retrospective follow-up study of the impact of genetic counselling on parental reproduction following the birth of a Down syndrome child. Clin Genet 1982; 21: 7-13.

${ }^{18}$ Boon AR. Tetralogy of Fallot-the effect on the family. Br J Soc Prev Med 1972; 26: 263-8.
${ }^{19}$ Smith JD. Down's syndrome, amniocentesis, and abortion: prevention or elimination? Mental Retardation 1981 (Feb); 8-11.

${ }^{20}$ Rynders JE, Spiker D, Horrabin JM. Underestimating the educability of DS children: examination of methodological problems in recent literature. Am J Ment Defic 1978; 82: 440-8.

\section{The XIth International Scientific Meeting of the International Epidemiological Association}

Helsinki, Finland, 8-13 August 1987

Main theme:

Sub-themes:

\section{EPIDEMIOLOGY AND HEALTH PROMOTION}

Health for all by the year 2000; national policies, general strategies, and assessment of prevention and health promotion

Fundamental aspects of epidemiology: methodology, ethics, etc

Communicable disease epidemiology and control

Epidemiology of non-communicable diseases and functional limitations

Non-communicable disease prevention and health promotion

Health services research

Teaching and training of epidemiology

Further information: IEA-Congress Secretariat

PO Box 189

SF-00171 HELSINKI

Finland 